\title{
Humidity as an aversive stimulus in learning in Drosophila melanogaster
}

\author{
ÉRIC LE BOURG \\ CNRS and Université Paul-Sabatier, Toulouse, France
}

\begin{abstract}
The learned suppression of photopositive tendencies was studied at the individual level in young flies of both sexes. In a T-maze, flies had to choose between an arm leading to a lighted vial associated with an aversive stimulus (i.e., a solution of quinine chlorhydrate deposited on a filter paper in the vial) and another arm leading to a darkened vial free of quinine. The present experiments were carried out to determine the roles of quinine and relative humidity in the maze. The flies avoided the lighted vial containing quinine even if they had no tarsal contact with quinine, and this result was not due to any odor of quinine. Subsequent experiments showed that relative humidity in the lighted vial, and probably in the arm leading to it, was an aversive stimulus, which partly explains why the flies avoided the lighted vial. However, in conditions in which the flies had tarsal contact with water or quinine it was confirmed that flies trained with quinine have higher avoidance scores than those trained with water only. Moreover, individual aversion to humidity was not correlated with the individual avoidance score: At similar levels of motivation (i.e., similar levels of aversion to humidity), some flies learn to avoid the lighted vial containing quinine whereas others do not. All these results show that, in addition to quinine, humidity is an unconditioned aversive stimulus in our paradigm and thus needs to be tightly controlled in experiments of conditioned avoidance.
\end{abstract}

Learning can be defined as a behavioral change linked to previous individual experience. Animal models are useful in the study of learning, and the fruit fly Drosophila melanogaster has been used for 30 years to this end (see Le Bourg \& Buecher, 2002, for a review).

Establishing paradigms for the study of learning is not an easy task and requires, in addition to replicability, accurate control and characterization of the stimuli driving the animal's response. The present article is an attempt to offer a suitable characterization of the stimuli involved in a recently described paradigm (Le Bourg \& Buecher, 2002).

This paradigm consists in using a T-maze with one darkened and one illuminated arm to train individual $D$. melanogaster flies to suppress their natural positive phototactic tendency and choose the darkened arm preferentially. The lighted arm leads to a lighted vial containing a filter paper wetted with an aversive quinine solution, whereas the darkened arm leads to a darkened vial with no aversive stimulus. Over a 16-trial training session, young flies of both sexes increase their tendency to choose the darkened vial when the lighted vial is wetted with quinine. By contrast, most flies tested with a dry lighted vial do not increase their tendency to avoid this vial (Le Bourg \& Buecher, 2002).

Many thanks are due Martin Giurfa for his helpful comments on previous versions of this article. Correspondence concerning this article should be addressed to É. Le Bourg, Centre de Recherches sur la Cognition Animale, UMR CNRS 5169, Université Paul-Sabatier, 118 route de Narbonne, 31062 Toulouse Cedex 4, France (e-mail: lebourg@cict.fr).
The procedure has several advantages. First, it does not require any preparation of flies before training, such as fasting (as is the case with conditioned inhibition of the proboscis-extension response (PER; see, e.g., Brigui, Le Bourg, \& Médioni, 1990). Second, it yields results quickly: A 16-trial training session takes about $15 \mathrm{~min}$ for each young fly. Third, the procedure does not require sophisticated devices that may be unavailable to many researchers (e.g., the flight simulator used to study visual learning in D. melanogaster; see Wolf \& Heisenberg, 1991). In such conditions, our paradigm could be of help in the study of learning in flies, provided that the increased avoidance of the lighted vial over trials is due to learning and not to other causes, such as sensitization to the stimuli encountered by the flies during training or avoidance based on the detection of stimuli at the choice point.

In our paradigm, it could be argued that the lighted arm provides a conditioned stimulus (CS - light) associated with an aversive unconditioned stimulus (US- the taste of quinine) whereas the darkened arm is not associated with an aversive stimulus. Flies would thus learn a Pavlovian association between stimuli (a CS-US association). However, since flies are naturally photopositive, it seems difficult to consider light as a CS - that is, as a neutral stimulus. This paradigm could then be assimilated to the conditioned inhibition of a reflex (as is the case with PER conditioned inhibition in fasting flies; see, e.g., Brigui et al., 1990), because flies would learn to inhibit a response toward a meaningful stimulus (i.e., light). However, the main difference between PER conditioned inhibition and the inhibition induced in our paradigm is 
that the former involves appetitive motivation whereas the latter does not. Hunger does not decrease unless it is satisfied, whereas positive phototaxis may spontaneously decrease when the duration of exposure to light increases (Médioni, 1958). It is possible, however, that flies make operant associations between a response and one or several stimuli (e.g., flies turn away from the light to avoid quinine) in our paradigm.

For unknown reasons, water is also an aversive stimulus in our paradigm; this is evident in flies' increased tendency over a 16-trial training session to choose the darkened vial when the lighted vial is wetted only with water. However, avoidance scores are higher when the lighted vial is wetted with quinine solution than when it is wetted with water only. Furthermore, when flies can move freely between two connected vials - one containing a piece of dry filter paper and the other a piece of filter paper wetted with water only - they spend more time in the dry vial, which indicates that the wet filter paper is aversive (Le Bourg \& Buecher, 2002). This result is similar to that of Sayeed and Benzer (1996), who reported that flies tested en masse prefer an environment with 3\% relative humidity over one with $99 \%$ relative humidity. All these results suggest that water is an aversive stimulus. Thus, the suppression of positive phototaxis in our paradigm may be due to the aversive quinine taste or to the combined action of quinine taste and water. The latter could be perceived by tarsal chemoreceptors when the fly reaches the wetted filter paper, but also by antennal hygroreceptors (Sayeed \& Benzer, 1996) in the presence of higher relative humidity in the lighted arm.

These assumptions help us to understand the results of a previous experiment on extinction in which our paradigm was used (Le Bourg \& Buecher, 2002, Experiment 3). After reaching a criterion of acquisition (i.e., reaching the darkened vial in three out of four trials) during training with a lighted vial containing quinine, flies were subjected to an extinction procedure in which this vial was replaced by one containing a dry filter paper. Instead of showing progressive extinction, flies chose the lighted vial from the very first extinction trial. By contrast, flies from control groups that were presented with a lighted vial containing either distilled water or quinine continued to choose the dark vial. Together, these results show that the absence of humidity is sufficient to disrupt the avoidance of the lighted vial and, thus, that the avoidance of the lighted vial was based not only on a conditioned avoidance of quinine but also on an unconditioned avoidance of humidity. Therefore, this experiment did not demonstrate the existence of an extinction process, because what was expected was a weakening of the conditioned response, at least in the first extinction trial, rather than an abrupt decline.

Avoidance of the lighted vial is greater when the vial is wetted with a quinine solution than when it is wetted with water only (Le Bourg, 2004; Le Bourg \& Buecher, 2002). Water and quinine could thus both be aversive stimuli, water less so than quinine. However, it could be argued that flies perceive an odor of quinine, if it indeed exists, and that this odor is repellent. It could also be argued that there is no association between a behavior (positive phototaxis) and an aversive stimulus (quinine) but only a nonassociative sensitization to quinine - that is, a decrease of the positive phototactic behavior due solely to repeated exposure to quinine. Quinine has been used for decades in learning studies with D. melanogaster because of its bitter taste (see, e.g., Brigui et al., 1990; Quinn, Harris, $\&$ Benzer, 1974). It does not seem to induce sensitization (Ackerman \& Siegel, 1986), and no effect of its possible odor has been reported. However, it is useful to verify whether sensitization to quinine and its possible odor has any effect in our procedure.

Our previous results raise questions about the precise role of water and humidity in our paradigm and call for new experiments allowing their importance in this context to be specified. Here, a series of experiments conceived to this end is presented. In the first experiment, the "extinction" experiment reported above (Le Bourg \& Buecher, 2002, Experiment 3) was replicated using flies of different ages. In the second experiment, I tested whether humidity in the absence of tarsal contact with quinine was sufficient to suppress the positive phototactic tendency of flies. The third experiment was a test of whether a possible quinine odor was more efficient than humidity in suppressing the positive phototactic tendency of flies. The fourth experiment was conducted to test whether preexposure to quinine modifies avoidance of the lighted vial wetted with quinine; that is, whether habituation to quinine has an effect on subsequent avoidance of the lighted vial. Finally, in the fifth experiment I tested whether sensitization to humidity could explain the increased avoidance of the lighted vial and whether individual aversion to humidity is correlated with the individual avoidance score.

\section{GENERAL METHOD}

The procedure has been described in detail in Le Bourg and Buecher (2002) and is summarized below.

\section{General Learning Procedure}

Individual flies had to make successive choices between a lighted arm and a darkened arm in a T-maze (Figure 1). Flies choosing the lighted arm reached a lighted vial, where they could be exposed to an aversive stimulus. Two transparent vials were set vertically at the exit of the arms of the T-maze. The lighted vial was internally covered with white filter paper except at its opening and at the opposite end. Depending on the experiment (see below), the filter paper in the lighted vial was dry or wetted with either a $10^{-1} \mathrm{M}$ solution of quinine hydrochloride (Fluka 22630) or distilled water. The darkened vial, free of filter paper, was inserted into another, opaque gray vial that prevented light from entering the darkened vial. A syringe containing a single fly was connected to the entrance of the maze, which was drilled into a block of opaque gray plastic. The width of the arm was $2 \mathrm{~mm}$ and the height $1.4 \mathrm{~mm}$, which prevented the fly from walking on the walls. The ceiling was made of transparent Plexiglas partially covered with a red filter. Flies are nearly blind to red light, so the subjects could not perceive the light transmitted through the ceiling; nevertheless, the experimenter could observe each fly while it crossed the maze. The temperature of the experimental room was $25^{\circ}$ $\pm 1^{\circ} \mathrm{C}$; this room was lit only with an optical fiber above the maze. 
TOP VIEW

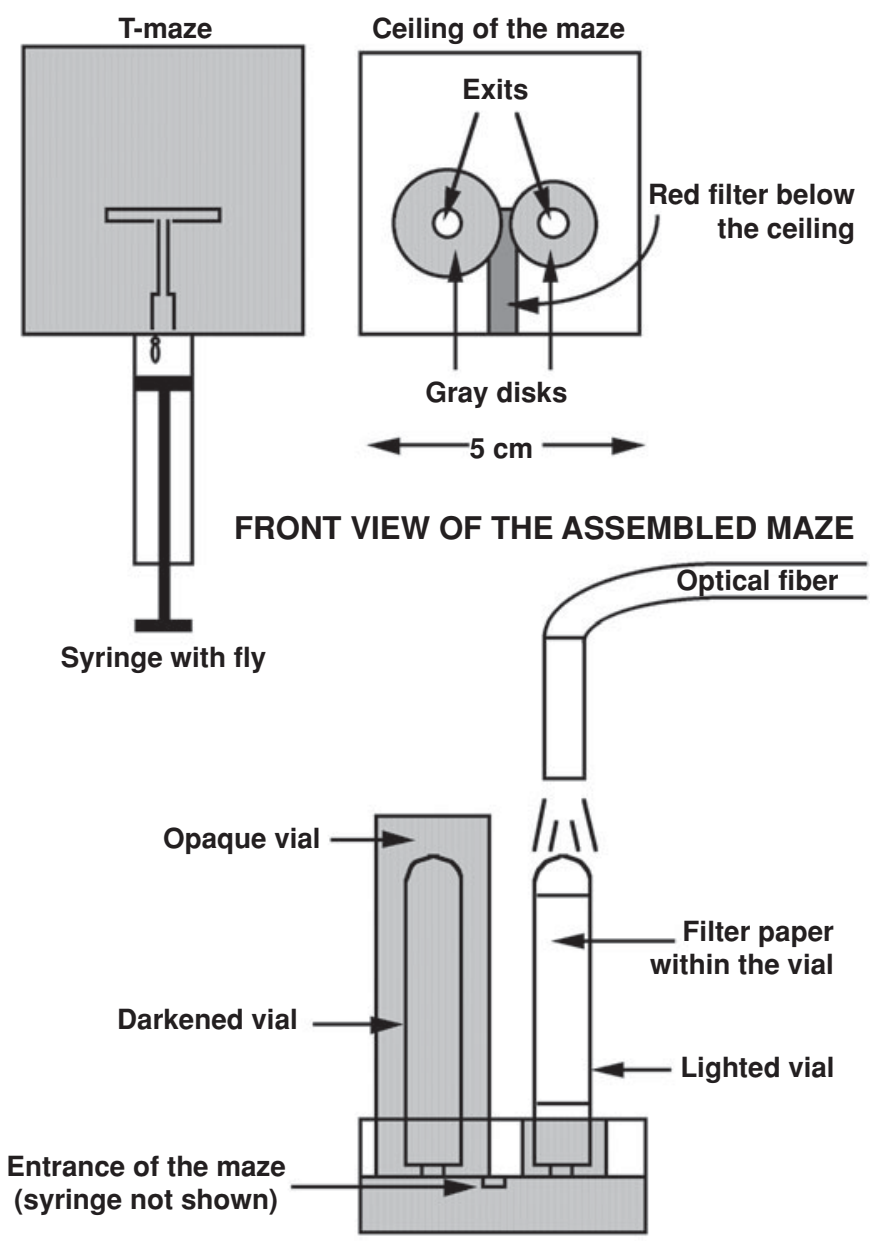

Figure 1. Schematic diagram of the apparatus used in the learning task, showing both top and front views. The figure shows the ceiling of the maze with the lighted vial set on the right exit. Another ceiling, symmetrical to that shown in the figure, was built with the lighted vial set on the left exit. From "Learned Suppression of Photopositive Tendencies in Drosophila melanogaster," by E. Le Bourg \& C. Buecher, 2002, Animal Learning \& Behavior, 30, p. 332. Copyright 2002 by The Psychonomic Society. Reprinted by permission.)

Each tested fly was transferred without anesthesia to the syringe and gently pushed to the start of the maze, where it made a choice between the two arms. If the fly returned to the start of the maze before reaching one of the vials, it was allowed to resume the trial. A fly entering the lighted vial was considered photopositive, whereas a fly entering the darkened vial was considered photonegative. The trial ended when the fly reached a vial. Flies that went into the darkened vial on the very first trial were discarded, which means that only those showing positive phototaxis on the first trial were trained, since the task consisted in suppressing this spontaneous tendency. When the fly reached a vial, this vial was separated from the maze and the fly was transferred to the syringe without anesthesia. The fly could walk on the filter paper during the time interval between its having reached the vial and its transfer to the syringe (usually 10 $15 \mathrm{sec}$ ), which means that the intertrial interval was about $30 \mathrm{sec}$. After transfer to the syringe, a new trial was run. Sixteen training trials were run in the training session. The lighted vial was on the right side of the maze $(\mathrm{R})$ for half of the trials, and a double alternation procedure (RRLLRRLLRRLLRRLL or LLRRLLRRLLRRLLRR) was used throughout the experiment. Two mazes were used: one with the ceiling designed for placement of the lighted vial on the right side and the other designed for placement on the left side. After each fly was tested, the mazes and ceilings were cleaned with distilled water and dried; the darkened and lighted vials were discarded, and new ones were used for the next fly. The experiments involved both sexes and were performed from about 09:00 to 17:00. The procedures of Experiments 1-4 are derived from this general procedure.

\section{Flies}

The experimental flies were adult males and females of the wild strain Meyzieu. This strain is maintained by mass mating on the standard medium (agar, sugar, corn meal, and dead yeast) enriched with live yeast. The experimental flies were obtained as follows: Eggs laid during a $15-\mathrm{h}$ period by 50 pairs of 5-day-old flies were transferred in batches of 25 to $80-\mathrm{ml}$ glass vials containing the medium described above. Upon emergence, flies with 9-10 days of 
preimaginal development were transferred under ether anesthesia, in same-sex groups of 15, to 20-ml polystyrene vials (Model 5111, Polylabo, France) containing the standard medium with a drop of live yeast. Vials were replaced twice a week. The flies spent their lives in an incubator (Maxi-Artic, Jouan; spektra.co.id/products/ prod_frame.htm); the rearing temperature was $25^{\circ} \pm 0.5^{\circ} \mathrm{C}$, and the photophase lasted from 08:00 to 20:00, illumination being provided by a fluorescent lamp.

\section{Statistical Data}

The following variables were computed: (1) percentage of flies reaching the lighted vial on the first trial, (2) number of photonegative choices (i.e., choice of the darkened vial) in four blocks of four trials, (3) time interval between the first and the last trial, and (4) number of trials during which the flies returned at least once to the start of the maze. This number may thus vary between 0 (no return observed) and 16 (at least one return on each trial).

The first variable reflects the initial phototactic tendency, following a previous study on phototaxis (Le Bourg \& Badia, 1995). Variables 2-4 were computed for flies that completed the 16-trial training. A variation in the number of photonegative choices during the four blocks, hereafter called the avoidance score, is a reflection of the tendency toward increased avoidance of the lighted vial. The time to complete training could depend on the presence of an aversive stimulus in the lighted vial (a log transformation of time was used in all analyses), because more time is required when the lighted vial is wetted with quinine than when it is dry (see Experiment 2 of Le Bourg \& Buecher, 2002). Similarly, the number of returns to the start of the maze could vary with the presence or absence of an aversive stimulus in the lighted vial and thus could reflect the behavior of the flies before they chose between the two vials (see Experiment 3 of Le Bourg \& Buecher, 2002).

Dichotomized data (Variable 1) were analyzed with a logistic model (Data Desk 6.1, Data Description Inc., available from www .datadesk.com). A logistic model is a generalized linear model fit to binary data. As the classical ANOVA, a logistic model is used to compute main effects of various factors (e.g., sex) as well as interactions between them.

\section{EXPERIMENT 1}

A previous experiment (Le Bourg \& Buecher, 2002) has shown that young flies subjected to an extinction procedure after having reached an acquisition criterion chose the lighted vial from the very first extinction trial (during extinction, both the lighted and the darkened vials were dry). This result could indicate that the absence of humidity is sufficient to disrupt the previously learned association or that humidity, detected at the choice point, is an unconditioned aversive stimulus, which would at least partly explain the avoidance of the lighted vial during training. To confirm these results, it was initially decided that several age groups would be used, rather than only young flies, because a previous study of the conditioned inhibition of PER showed that age had some effect on extinction (Brigui et al., 1990). However, the study of aging is not of central interest in the context of this article.

\section{Method}

The present experiment replicates the extinction procedure with males and females of different ages $(1,2,3,4,5,6$, and 7 weeks old), the exact age being the number of weeks \pm 2 days. The flies were subjected to a training procedure in which a lighted vial containing a paper wetted with a quinine solution was used on each trial up to a criterion of acquisition (three photonegative choices in 4 successive trials) or to the 16th training trial if the criterion was not reached. Half of the flies reaching the criterion were then subjected to 16 extinction trials, during which the filter paper internally covering the lighted vial was dry (extinction group). The other half were subjected to 16 more training trials (overtraining group). Ten flies of each sex in each age group and each reinforcement group (extinction vs. overtraining) completed the experiment (i.e., $N=280$ ).

\section{Results and Discussion}

The phototactic tendency on the first training trial was analyzed with a logistic model for dichotomized data using the 613 flies that had completed the first training trial. The females were more photopositive $(85.08 \%, n=248)$ than the males $[74.52 \%, n=365 ; F(1,599)=9.47, p=$ $.0022]$. Age and its interaction with sex had no significant effect on phototaxis ( $F$ s close to 1 , data not shown).

An ANOVA with three independent factors (sex, age, and extinction/overtraining procedure) and one dependent factor (blocks of trials) was used to analyze the 16 postacquisition trials. As was expected, flies subjected to extinction avoided the lighted vial less than did those subjected to overtraining [Figure 2A; $F(1,252)=526.40, p<.0001$ ] Neither the sex and age effects nor their interaction were significant $(F$ s close to 1$)$. The significant sex $\times$ extinction procedure interaction $[F(1,252)=4.39, p=.0371]$ showed that the males had slightly higher scores than the females if they were overtrained and slightly lower scores if they were subjected to extinction. Figure 2A shows that no age effect was observed in overtrained flies, whereas the number of photonegative choices decreased in older flies subjected to extinction; however, this pattern was insufficient to give rise to a significant age $\times$ extinction procedure interaction $[F(6,252)=1.93, p=.0761]$. The number of photonegative choices increased slightly with blocks of trials. Figure 2B illustrates the results for the 1 -week-old group $[F(3,756)=31.96, p<.0001]$, but the block $\times$ age interaction showed that this pattern was observed less in older flies $[F(18,756)=1.79, p=$ $.0221]$. The second-order interaction between sex, extinction procedure, and blocks showed that the females had higher scores than the males on some blocks if they were subjected to extinction and lower scores if they were subjected to overtraining $[F(3,756)=5.53, p=.0009]$. No other interaction was significant ( $F$ s close to 1$)$.

It was of interest to test whether or not the first nonavoidance during the extinction/overtraining phase (i.e., the first trial on which the fly reached the lighted vial) varied with age, sex, or the extinction/overtraining procedure. It was impossible to compute an ANOVA due to the very large number of ties - that is, of flies reaching the lighted vial in the first trial. The flies were thus dichotomized in two groups: those reaching the lighted vial in the first trial and those reaching it in any other trial (all flies went to the lighted vial at least once). This variable was analyzed with a logistic model using the 280 flies that completed the experiment; it was necessary to pool the two oldest age groups due to an empty cell (all 6-week-old males subjected to extinction reached the lighted vial in the first 

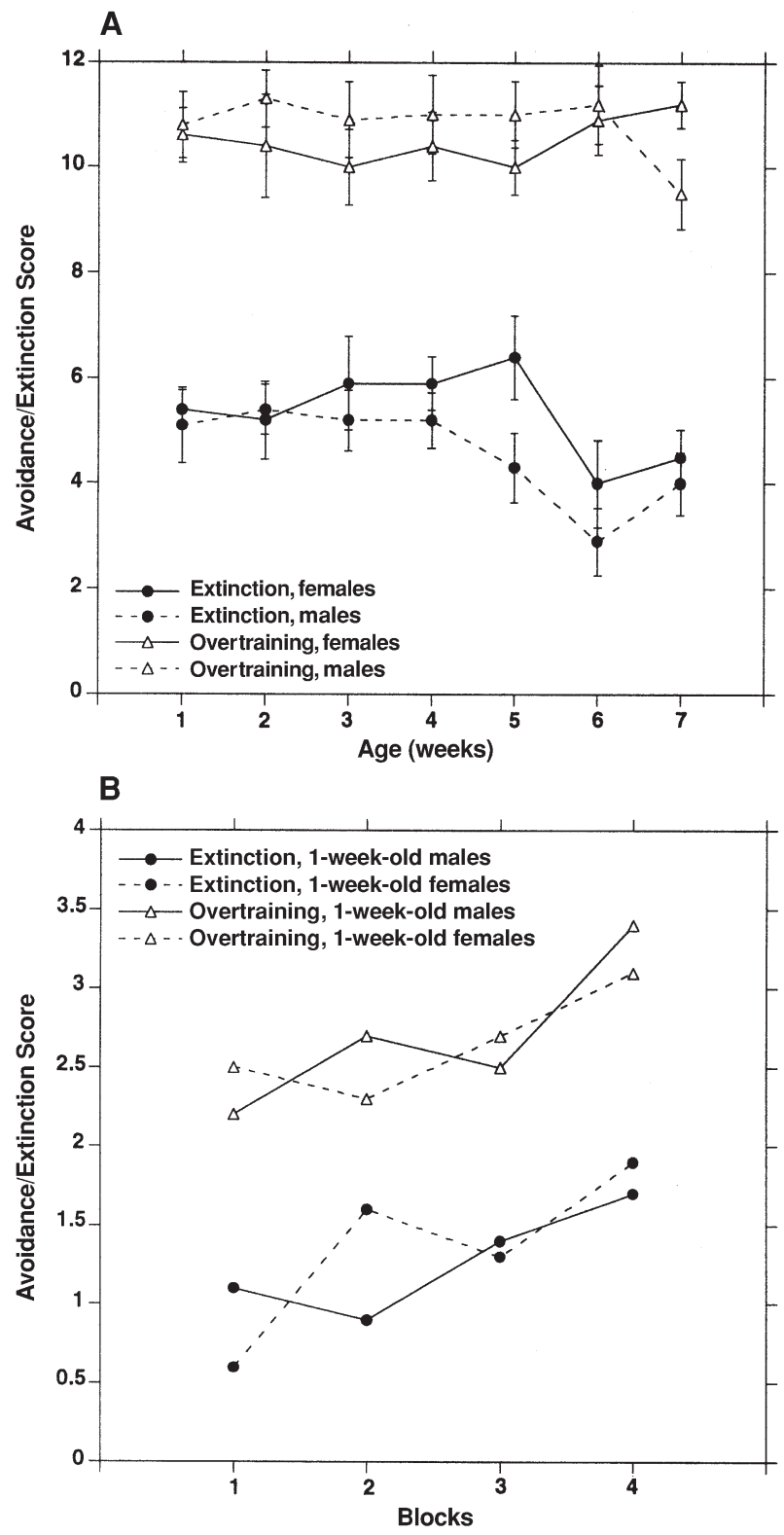

Figure 2. Results of Experiment 1. (A) Number of photonegative choices (avoidance or extinction score $\pm S E M$ ) as a function of age. The flies were subjected either to extinction or to overtraining after reaching an acquisition criterion. Each point is the mean of 10 males or 10 females, and the avoidance/extinction score can vary between 0 and 16. (B) Number of photonegative choices (avoidance or extinction score) as a function of blocks of trials in 1-week-old flies. This figure is included only to illustrate the block effect. For each block, the score can vary between 0 and 4. Each point represents the mean of 10 males or 10 females.

trial). The flies subjected to extinction (85.71\% of which reached the lighted vial in the first trial) chose the lighted vial more rapidly than did the overtrained flies $[55.00 \%$; $F(1,256)=21.32 p<.0001]$. Age and sex effects and all interactions were not significant ( $F$ s close to 1 , data not shown). These results confirm that the flies chose between the lighted and darkened arms by relying not only on light differences between them but also on humidity cues.

The main result of this experiment is that flies of various ages that have previously reached a criterion of avoidance of the lighted vial containing quinine no longer avoid the lighted vial when it is dry, as has been observed in young flies (Le Bourg \& Buecher, 2002). This result is unexpected if flies have actually learned that the lighted vial is associated with quinine, because they should avoid the lighted vial at least in the first extinction trials. This result thus means that the flies did not simply make an association between the lighted vial and quinine. Water and humidity could also have been involved. Removing the quinine solution from the lighted vial results in removal of water from the same vial, as well as removal of humidity and quinine odor, if any, from the lighted arm. It has been shown that water is an aversive stimulus (Le Bourg $\&$ Buecher, 2002). In addition, humidity could act as a discriminative stimulus as the fly anticipates the aversive tarsal contact with quinine and water. The flies could detect with their hygroreceptors that the lighted arm was humid and learn to expect quinine if it was associated with both light and humidity: When flies are subjected to extinction, the inappropriate context (absence of humidity) would lead to nonavoidance of the lighted vial. This explanation, if correct, is based on the assumption that water plays two roles in the context of our paradigm: It acts as an aversive stimulus perceived by tarsal chemoreceptors in the lighted vial, and it is also perceived by the hygroreceptors as humidity in the lighted arm of the maze. In the latter case, humidity could be a signal of the aversive stimuli present in the lighted vial (water and quinine). In addition, humidity itself could be an aversive stimulus, since flies may be unwilling to enter humid areas. Finally, quinine odor, if it indeed exists, could also be an aversive stimulus, preventing flies from entering areas that contain it.

\section{EXPERIMENT 2}

The previous experiment has shown that removing quinine from the lighted vial is sufficient to allow flies of all ages to recover their photopositive tendency immediately after they have suppressed it. To better understand the roles of humidity and quinine odor, in the present experiment I tested whether humidity and quinine odor, without tarsal contact with water and quinine, were sufficient to suppress the positive phototactic tendency of flies. Thus, this experiment was a test of whether humidity and quinine odor could be aversive stimuli.

\section{Method}

Young flies ( $7 \pm 2$ days old $)$ were subjected to 16 trials of training with a lighted vial. For half of the flies (quinine group), the vial contained a filter paper wetted with a quinine solution as an aversive stimulus. For the other half (dry group), the vial contained a dry paper. For half of the flies in the quinine group and half in the dry group, a 1-ml Gilson blue tip cut $20 \mathrm{~mm}$ from the wide end was inserted into both the lighted and the darkened vials. A plastic grid, heat-soldered to the bottom of the cut tip, prevented the flies from reaching the paper but allowed humidity and odors to reach the flies. 
Thus, some of the flies had tarsal contact with the quinine whereas others were subjected only to humidity and the odor of quinine, if it indeed exists. After each fly was tested, the modified tips were cleaned with distilled water and dried. Five flies of each sex completed the experiment for each group (quinine-tip, quinine-no tip, dry-tip, dry-no tip); that is, $N=40$.

\section{Results and Discussion}

The phototactic tendency in the first training trial was analyzed with a logistic model for dichotomized data using the 58 flies that completed the first training trial. A logistic model cannot be computed if there is an empty cell, and this was the case here (no male of the dry-tip group was photonegative). Thus, the results of the males (77.42\% photopositive, $n=31)$ and the females $(88.89 \%$ photopositive, $n=27$ ) were pooled. The presence or absence of quinine (quinine vs. dry condition) and of the tip (tip vs. no-tip condition) had no effect on phototaxis, and the interaction between these two factors was not significant ( $F$ s close to 1 , data not shown).

An ANOVA with three independent factors - reinforcement (quinine vs. dry), presence of tip (tip vs. no tip), and sex - and one dependent factor (blocks of trials) showed that the flies trained with quinine made more photonegative choices than did those trained under the dry condition [Figure 3; $F(1,32)=76.33, p<.0001]$. The number of photonegative choices increased with blocks of trials $[F(3,96)=11.32, p<.0001]$, and the nearly significant interaction between reinforcement and blocks showed that

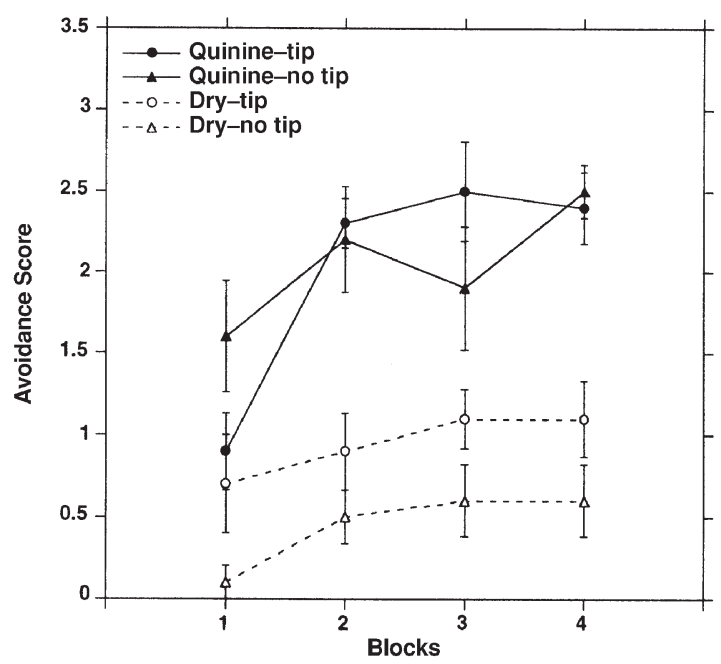

Figure 3. Results of Experiment 2. Mean number of photonegative choices (avoidance score $\pm S E M$ ) as a function of blocks of 4 trials. For each block, the score can vary between 0 and 4 . One half of the flies were trained with a lighted vial containing a paper wetted with a quinine solution as an aversive stimulus on each of 16 trials (quinine). The other half were trained with a lighted vial containing a dry paper (dry). One half of the flies in each of these groups were trained with a modified 1-ml Gilson tip in the lighted and darkened vials; tarsal contact with quinine and water was prevented (tip) but the passage of humidity and odors was allowed. The other half were trained without this tip (no tip). Each point represents the mean of 5 males and 5 females. this increase is attributable mainly to the flies trained with quinine $[F(3,96)=2.43, p=.0702]$. Sex, presence of the tip effects, and all interactions were not significant except for the third-order interaction between reinforcement, presence of tip, sex, and blocks, which was due mainly to a high score on the first block of the females trained under the dry condition with the tip $[F(3,96)=3.73, p=$ .0137].

In another experiment, rather than a Gilson tip, a small, transparent polystyrene vial was used (length $41 \mathrm{~mm}$, diameter $8 \mathrm{~mm}$ ), which was cut $9 \mathrm{~mm}$ from its open end and closed with a heat-soldered grid. This vial was inserted in the lighted vial only. Thus, the experiment was repeated with a polystyrene (rather than polypropylene) vial of a different size and color (transparent vs. blue) and with a different procedure (no polystyrene vial inserted into the darkened vial), to determine whether similar results can be obtained with slightly modified conditions. Similar results were indeed observed, except that the third-order interaction between reinforcement, presence of the tip, sex, and blocks was not significant (data not shown).

An ANOVA with three independent factors - reinforcement (quinine vs. dry), presence of the tip (tip vs. no tip), and sex - showed that more time was needed to complete the 16 learning trials by the flies trained with quinine $[F(1,32)=15.18, p=.0005$; mean $\pm S E M$ for the flies trained with quinine, $15.20 \pm 0.83 \mathrm{~min}$; mean $\pm S E M$ for the flies trained in the dry condition, $11.60 \pm 0.66 \mathrm{~min}]$ and by the females $[F(1,32)=16.51, p<.0001$; mean for the females, $14.50 \pm 0.88 \mathrm{~min}$; mean for the males, $12.30 \pm 0.76 \mathrm{~min}]$. The flies trained with the tip also took more time $[F(1,32)=5.02, p=.032$; mean $\pm S E M$ for the flies trained with the tip, $14.35 \pm 0.78$ min; mean \pm $S E M$ for the flies trained with no tip, $12.45 \pm 0.88 \mathrm{~min}]$, but this effect reflects mainly that it was not easy to see the fly in the lighted vial when the blue tip was inserted into it and is thus meaningless. None of the interactions was significant. An ANOVA with three independent factors (reinforcement, presence of the tip, and sex) showed that the number of returns to the start of the maze was larger under the quinine condition $[F(1,32)=26.68, p<$ .0001 ; with quinine, $4.65 \pm 0.45$ returns; dry, $1.45 \pm 0.44$ returns], the other main factors and all interactions being nonsignificant. Not unexpectedly, the number of returns was positively correlated with time to complete training (computations using the log of times: $r=.72, p<$ $.0001)$. These results show that the flies trained with quinine needed more time to complete the experiment, partly because they made more returns to the start of the maze. Similar results have been observed when flies come into tarsal contact with quinine (Le Bourg \& Buecher, 2002), but the present experiment shows that this contact is not necessary.

This experiment thus shows that the flies trained with quinine attained high scores even if they did not come into tarsal contact with the quinine, because the presence of the tip had no effect on the observed scores. In other words, the flies perceived humidity or possibly a quinine odor, 
and this perception was sufficient to induce an increasing avoidance of the lighted vial over trials. Nevertheless, this experiment does not allow us to separate the effect of humidity from that of a possible quinine odor; the next experiment was performed to this end.

\section{EXPERIMENT 3}

\section{Method}

In order to separate the possible effect of the quinine odor from that of humidity, the previous experiment was repeated using the same methods except that one half of the flies were trained with a lighted vial containing a paper wetted with distilled water (water groups) rather than with a dry vial. The flies trained with no tip had tarsal contact with quinine or water, whereas those trained with a tip were subjected only to humidity or to quinine odor, if it indeed exists. Obviously, the flies trained with quinine were also subjected to the water stimulus and those trained with quinine odor, if it exists, were also subjected to humidity, because quinine dissolves in water. Therefore, it would be concluded that quinine or its odor have an effect on avoidance scores if flies trained with these stimuli attained higher scores than those trained with water or humidity only. Five young flies ( $7 \pm 2$ days old) of each sex completed the experiment in each group (quinine-tip, quinine-no tip, water-tip, water-no tip; that is, $N=40)$.

\section{Results and Discussion}

The phototactic tendency in the first training trial was analyzed with a logistic model for dichotomized data using the 64 flies that completed the first training trial. Due to an empty cell (all flies being photopositive), the results of the males ( $84.38 \%$ photopositive, $n=32$ ) and the females ( $71.88 \%$ photopositive, $n=32$ ) were pooled. The presence of quinine or of tip had no effect on phototaxis, and their interaction was not significant $(F$ s close to 1 , data not shown).

An ANOVA with three independent factors-reinforcement (quinine vs. water), presence of the tip (tip vs. no-tip), and sex - and one dependent factor (blocks of trials) showed that the males had a slightly higher score on avoidance of the lighted vial than did the females $[F(1,32)=4.20, p=.0486$; mean for the males, $7.75 \pm$ 0.47 ; mean for the females, $6.30 \pm 0.50]$. The number of photonegative choices increased with blocks $[F(3,96)=$ $18.36, p<.0001]$, and the only significant interactionthat between reinforcement, sex, and blocks $[F(3,96)=$ $2.81, p=.0437]$ - showed no difference between the females in the quinine group and those in the water group in the first block (mean: 0.9 photonegative choice), whereas the males trained with water ( 0.8 photonegative choice) had a slightly lower score than did those trained with quinine (1.4 photonegative choice). Clearly enough, this effect was weak. The reinforcement and presence-of-tip effects (Figure 4A) were not significant.

An ANOVA with three independent factors (reinforcement, presence of the tip, and sex) showed only that the time to complete the 16 learning trials was longer for the flies trained with a tip $[F(1,32)=5.67, p=.0234]$, which is a meaningless effect, as was discussed in Experiment 2. An ANOVA with three independent factors (reinforcement, presence of the tip, and sex) showed no significant effect on number of returns to the start of the maze (data not shown).
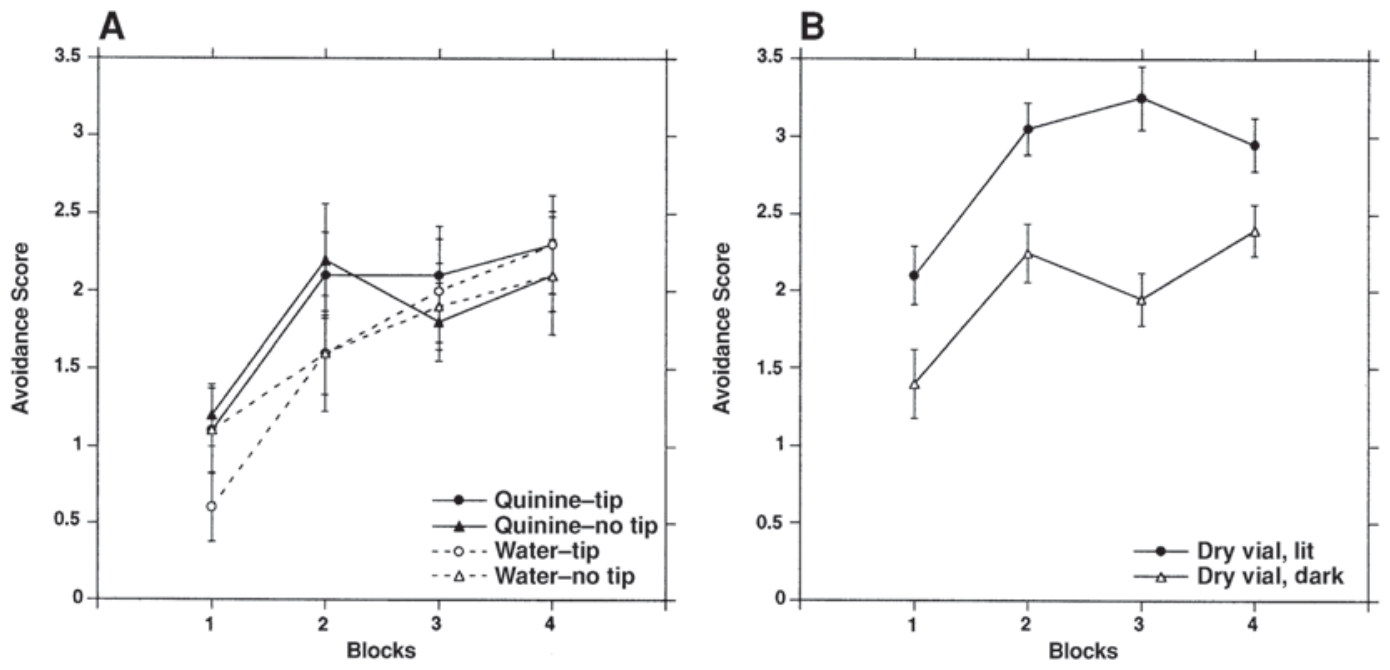

Figure 4. Results of Experiment 3. (A) Mean number of photonegative choices (avoidance score $\pm S E M$ ) as a function of blocks of 4 trials. One half of the flies were trained with a lighted vial containing a paper wetted with a quinine solution as an aversive stimulus on each of 16 trials (quinine). The other half were trained with a lighted vial containing a paper wetted with distilled water (water). One half of the flies in each of these groups were trained with a modified 1-ml Gilson tip in the lighted and darkened vials (tip), and the other half were trained without this tip (no tip). Each point represents the mean of 5 males and 5 females. (B) Mean number of photonegative choices (avoidance score $\pm S E M$ ) as a function of blocks of 4 trials. The flies were trained with a lighted vial containing a paper wetted with a quinine solution as an aversive stimulus on each of 16 trials and with no tip. For one half of the flies, the opaque vial (see Figure 1) was removed (dry vial, lit) after the first trial, and for the other half it was not removed (dry vial, dark). Each point represents the mean of 10 males and 10 females. 
This experiment thus shows that the flies trained with quinine and those trained with water attained similarly high avoidance scores even if they did not come into tarsal contact with quinine, because the presence of the tip had no effect on the scores. Olfactory cues from quinine do not drive the choices of flies because adding quinine does not increase the scores above those of flies tested with humidity only. This means that the presence of humidity in the lighted vial is necessary and sufficient to cause photonegative choices to increase during experiments. In other words, flies perceive the humidity coming from the lighted vial and increasingly avoid this vial over trials. Forcing flies to walk on wet paper to avoid the lighted vial is therefore unnecessary to achieve avoidance. However, this does not mean that quinine is not a negative reinforcer because it increases avoidance scores, as previously shown (Le Bourg, 2004; Le Bourg \& Buecher, 2002) and discussed above.

Humidity was not expected to be aversive because flies live in rather moist environments, such as on rotten fruits, and survive only for a few hours in dry air (see, e.g., Minois \& Le Bourg, 1999). In order to verify the aversive role of humidity, an additional experiment was performed. Forty 1-week-old flies of both sexes were trained under the quinine-no-tip condition, but for half of them the opaque vial covering the darkened vial was removed after the first trial, making the formerly darkened, quininefree vial as bright as the vial containing quinine. Because both vials were now illuminated, the flies were expected to choose the vial with no quinine (i.e., the vial that was darkened in the first trial) very rapidly if humidity is an aversive stimulus. Indeed, flies at the T-choice point now had to choose between a dry arm leading to a dry vial and a humid arm leading to a quinine-filled vial. Since both arms were illuminated, there was no conflict between positive phototaxis and the avoidance of a humid area, and flies are thus expected to prefer the dry arm. An ANOVA with two independent factors (removal or nonremoval of the opaque vial and sex) and one dependent factor (blocks of trials) showed that removing the opaque vial increased the number of choices of the vial containing no quinine [Figure 4B; $F(1,36)=27.43, p<.0001]$. The number of photonegative choices increased with blocks $[F(3,108)=$ $13.55, p<.0001]$. The sex effect and all interactions were not significant. These results thus show that in the absence of darkness the flies strongly avoided the humid environment and thus the quinine vial. It could be argued that the flies have learned very rapidly to avoid the quinine vial, but an analysis of the time to complete the 16 training trials and the number of returns made by the flies during training rules out this hypothesis. An ANOVA with two independent factors (removal or nonremoval of the opaque vial and sex) showed that the time to complete the 16 learning trials was shorter with the opaque vial removed $[F(1,36)=24.42, p<.0001$; the means were $9.90 \pm 0.38 \mathrm{~min}$ and $14.30 \pm 0.93 \mathrm{~min}$ with removal and with nonremoval of the opaque vial, respectively], which seems to confirm that they went directly to the dry vial when it was lighted, as was the vial containing quinine.
The males $(11.00 \pm 0.60 \mathrm{~min})$ had shorter times than the females [13.20 $\pm 1.01 \mathrm{~min} ; F(1,36)=5.42, p=.0256]$, the interaction between the two factors being nonsignificant. Not unexpectedly, an ANOVA with two independent factors (removal or nonremoval of the opaque vial and sex) showed that the number of returns was much smaller when the two vials were illuminated $[F(1,36)=23.89$, $p<.0001$; the means were $1.20 \pm 0.19$ returns and 3.60 \pm 0.46 returns with removal and with nonremoval of the opaque vial, respectively], the sex effect and the interaction between the two factors being nonsignificant. Therefore, when the vial without quinine is as illuminated as the one containing quinine, flies go immediately and directly to the vial without quinine. Clearly, when flies must choose between a humid arm and a dry arm at the choice point, they choose the dry arm - that is, the vial without quinine. The results of this experiment are in accordance with those of a previous experiment that showed that flies moving freely between two connected vials - one containing a dry filter paper and the other a filter paper wetted with water only-spend more time in the dry vial (Le Bourg \& Buecher, 2002). Humidity is thus an aversive stimulus: When flies choose between a lighted dry arm leading to a dry vial and a lighted humid arm leading to a vial containing quinine, they choose the dry arm.

Quinine is also an aversive stimulus, as is shown by the fact that flies coming into contact with it have higher scores of avoidance of the lighted vial than do those trained with water only (Le Bourg, 2004; Le Bourg \& Buecher, 2002). Nevertheless, in those studies the effect of quinine on avoidance scores was weak in comparison with that of water. This effect is not significant in the quinine-no-tip and water-no-tip groups (see Figure 4A), which were subjected to the same conditions as those of the previously reported experiments. In Figure 4A, however, the sample size was small $(N=40)$ in comparison with, for instance, the sample sizes of Experiment 2 in Le Bourg $(2004 ; N \mathrm{~s}=$ 280 and 80). Even if this effect of quinine on avoidance scores is weak, it could be due to sensitization - that is, to a decrease of the positive phototactic behavior resulting only from repeated exposure to quinine. In the following experiment, this hypothesis is tested.

\section{EXPERIMENT 4}

It could be argued that the increase of avoidance scores in our paradigm is not due to an association between a behavior (suppressing positive phototaxis) and the resulting outcome (avoiding quinine), but that it results from sensitization to quinine - that is, repeated exposure to quinine would increase the avoidance response nonassociatively. Even if quinine does not seem to induce sensitization (Ackerman \& Siegel, 1986), it is useful to verify whether this is also the case in our procedure. Therefore, this experiment was conducted to test whether preexposure to quinine modifies avoidance scores. If avoidance of the lighted vial during training is due to sensitization to quinine, flies that are habituated to quinine after preexposure to it should avoid the lighted vial containing quinine less 
than do nonpreexposed flies. By contrast, if preexposure sensitizes flies to quinine, they should avoid the lighted vial more than nonpreexposed flies.

\section{Method}

In the afternoon preceding the day of experiment, preexposed flies were transferred to a vial containing the usual medium and a filter paper covering about $50 \%$ of its surface. This paper was wetted with quinine in the same ratio of quinine (by volume) to paper surface as was applied to the lighted vial used in the avoidance experiments. Control flies had no paper in their rearing vials. Therefore, for 1 night the flies could either walk on the filter paper wetted with quinine or try to avoid it. Young preexposed and control flies ( $7 \pm$ 2 days old) were subjected to training with a lighted vial containing a filter paper wetted with a quinine solution as an aversive stimulus on each of 16 trials. Ten flies of each sex completed the experiment for preexposed and control groups (i.e., $N=40$ ).

\section{Results and Discussion}

The phototactic tendency in the first training trial was analyzed with a logistic model for dichotomized data using the 60 flies that completed the first training trial. Sex, preexposure condition, and their interaction had no significant effect ( $F$ s close to 1, data not shown), and $66.67 \%$ of the flies were photopositive in the first trial. This percentage is lower than that found in previous experiments, and it was decided to replicate the experiment. In a second experiment carried out nearly 1 month after the first, the percentage of photopositive flies was $86.54 \%(n=52)$.

Since a replicate of the experiment was done, a replicate factor (random factor) was used in the ANOVA of avoidance scores. An ANOVA with three independent factors (replicate, preexposure, and sex) and one dependent factor (blocks of trials) showed that the replicate effect was significant $[F(1,72)=25.90, p<.0001$; see Figure 5A] and that all interactions involving the replicate factor were not significant. The number of photonegative choices increased with blocks $[F(3,3)=12.68, p=.0328]$, and the interaction between sex and blocks $[F(3,3)=18.33, p=$ $.0197]$ showed that the females had a slightly lower score than the males in the fourth block, whereas the contrary was true for the other blocks (data not shown). Sex and preexposure effects and the other interactions were not significant $(F$ s close to 1$)$. Very similar results were obtained when the replicate factor was removed from the analysis, except that the sex $\times$ blocks interaction was no longer significant. An ANOVA with three independent factors (replicate, preexposure, and sex) performed on time to complete the experiment showed effects for replicate and sex only, with the females being slower than the males (data not shown). The ANOVA performed on number of returns to the start of the maze showed only an effect of the replicate (data not shown). These results show that preexposure to quinine for several hours before the experiment had no effect on avoidance scores. It thus seems that flies do not avoid the lighted vial because they are sensitized to quinine during training, as was previously shown in another learning task (Ackerman \& Siegel, 1986).

The higher scores obtained when flies were trained with quinine rather than water (Experiment 2 of Le Bourg, 2004; Experiment 1 of Le Bourg \& Buecher, 2002) may
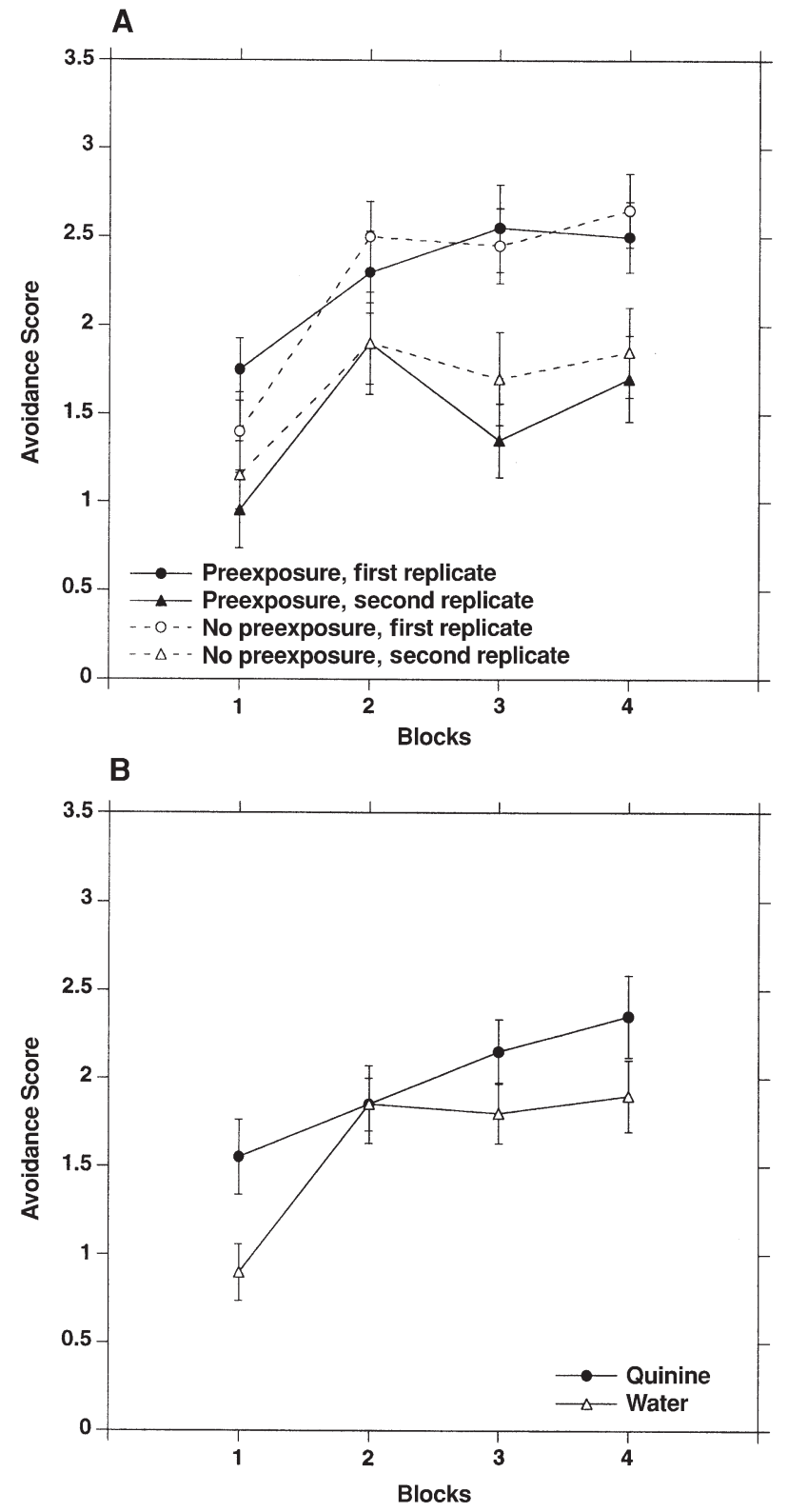

Figure 5. Results of Experiment 4. (A) Mean number of photonegative choices (avoidance score $\pm S E M$ ) as a function of blocks of 4 trials. For each block, the score can vary between 0 and 4 . The flies were trained with a lighted vial containing a paper wetted with a quinine solution as an aversive stimulus on each of 16 trials. One half of the flies were subjected to several hours of preexposure to quinine before the experiment (preexposure), whereas the other half were not (no preexposure). The figure reports the results of two replicates. Each point is the mean of 20 males and 20 females. (B) Mean number of photonegative choices (avoidance score $\pm S E M$ ) as a function of blocks of 4 trials. The flies were trained with a lighted vial containing a paper wetted either with a quinine solution or distilled water on each of 16 trials. Each point represents the mean of 10 males and 10 females.

therefore be due to an aversive effect of quinine that builds upon that of humidity. An experiment was thus conducted to verify that the avoidance scores of flies trained with quinine are indeed higher than those of flies trained with 
water. Young flies ( $7 \pm 2$ days old) were subjected to training with a lighted vial containing a filter paper wetted with either a quinine solution or distilled water as an aversive stimulus on each of 16 trials. Ten flies of each sex completed the experiment for each reinforcement group (i.e., $N=40$ ). A three-way ANOVA with two independent factors (reinforcement and sex) and one dependent factor (blocks of trials) showed that the flies attained higher scores when trained with quinine than when trained with water [Figure $5 \mathrm{~B} ; F(1,36)=4.48, p=.0414]$. The number of photonegative choices increased with blocks $[F(3,108)=10.33, p<.0001]$. The sex effect and all interactions were not significant. An ANOVA with two independent factors (reinforcement and sex) showed only that the flies trained with quinine needed more time to complete the experiment (data not shown). The ANOVA on the number of returns to the start of the maze showed that the flies trained with quinine had a marginally significant tendency to make more returns than did those trained with water (data not shown).

This experiment confirms that flies trained with quinine attain higher avoidance scores than those trained with water only. It thus can be argued that our paradigm makes it possible to observe an operant association between an aversive stimulus (i.e., quinine) and a behavior (i.e., avoidance of the vial containing quinine) and that flies do not avoid the lighted vial only because of its high relative humidity. Nevertheless, it could also be argued that even if quinine increases avoidance of the lighted vial, most of this avoidance is due to sensitization to humidity during training and not to learning. The next and final experiment tests this hypothesis.

\section{EXPERIMENT 5}

It could be argued that the increase of avoidance scores in our paradigm is not due to an association between a behavior (i.e., suppression of positive phototaxis) and the resulting outcome (i.e., avoidance of humidity and quinine), but that it results mainly from sensitization to humidity, as has been hypothesized for quinine. This hypothesis cannot be tested with the procedure used to test sensitization to quinine - that is, insertion of a paper wetted with distilled water into the rearing vial for 1 night - for two reasons. First, rearing vials have a 70\% relative humidity (Le Bourg \& Minois, 1999); adding a wet filter paper would probably not really increase this level. Second, Experiment 4 has shown that insertion of a paper wetted with a quinine solution into the rearing vial did not induce any sensitization to quinine. However, since the inserted paper was wet, it could be argued that this procedure did not induce any sensitization to humidity either. Thus, another procedure was preferred: Flies were subjected to a short exposure to humidity just before the experiment rather than overnight.

\section{Method}

Preexposure to humidity was obtained by subjecting flies to a spontaneous preference test between wet and dry filter papers using the procedure described in Le Bourg and Buecher (2002). Two 3-mL vials were internally covered with a filter paper up to the opening, a 1-mm space being left free of paper. One of the vials was wetted with $160 \mu \mathrm{L}$ of distilled water (the same ratio of quinine (by volume) to paper surface as was applied to the lighted vial used in the avoidance experiments), and the flies were individually placed in one of the vials, which was then connected to the other vial. The two connected vials were put on a table illuminated by an optical fiber, and immediately after a 5-min period the time spent in the dry vial was recorded. New vials were used for each fly. Immediately after this experiment, the flies were subjected to training with a lighted vial containing a filter paper wetted with a quinine solution as an aversive stimulus on each of 16 trials. A control group was not subjected to preexposure to humidity. Fifteen young flies ( $6 \pm 1$ days old $)$ of each sex completed the experiment for each group (preexposure to humidity and control; i.e., $N=60$ ).

Therefore, this experiment was conducted to test whether preexposure to humidity modifies avoidance scores. If avoidance of the lighted vial during training is due to sensitization to humidity, flies that are habituated to humidity after preexposure to it should avoid the lighted vial containing quinine, and thus humidity, less than control flies do. By contrast, if preexposure sensitizes flies to humidity, they should avoid the lighted vial more than control flies do. Finally, if the avoidance score reflects mainly learning ability rather than sensitization to humidity, preexposure to humidity should have no effect on avoidance scores.

This experiment also provides a test of whether or not the spontaneous preference for the dry vial is correlated with the avoidance score. Obviously, this computation involves only the 30 flies preexposed to humidity. If avoidance of the lighted vial during the 16 training trials is due not to learning but only to a spontaneous aversion to humidity, the time spent in the dry vial during preexposure to humidity should be highly positively correlated with the avoidance score. On the other hand, if there is no correlation it could be concluded that aversion to humidity is a prerequisite for learning to avoid the lighted vial, but that the avoidance score reflects mainly learning ability. In other words, with similar levels of motivationthat is, with the same aversion to humidity - some flies could learn to avoid the lighted vial whereas other flies could not.

\section{Results and Discussion}

The phototactic tendency in the first training trial was analyzed with a logistic model for dichotomized data using the 90 flies that completed the first training trial. Sex and preexposure condition had no significant effect ( $F$ s close to 1 , data not shown), and $71.11 \%$ of the flies were photopositive in the first trial. The significant sex $\times$ preexposure condition interaction $[F(1,86)=5.74, p=$ .0187] showed that the males preexposed to humidity were less photopositive than the control flies, whereas the contrary was observed for the females.

An ANOVA with two independent factors (preexposure to humidity and sex) and one dependent factor (blocks of trials) showed that the number of photonegative choices increased with blocks, as is usually observed $[F(3,168)=$ $11.90, p<.0001]$. Sex and preexposure effects and all interactions were not significant $(F$ s close to 1$)$. An ANOVA with two independent factors (preexposure and sex) performed on time to complete the experiment showed only that the females were slower than the males (data not shown). The ANOVA performed on number of returns to the start of the maze showed no significant effect (data not shown).

These results thus show that $5 \mathrm{~min}$ of preexposure to humidity before the experiment had no effect on avoid- 
ance scores. It could nevertheless be argued that during preexposure to humidity flies are in contact with the wet paper for too short a time to induce sensitization. If this were true, no sensitization should be observed during training either, because flies reaching the lighted vial wetted with quinine are in contact with the wet paper for only a few seconds.

During preexposure to humidity, the flies spent $221.53 \pm$ $7.60 \mathrm{sec}$ in the dry vial $[t$ test of the difference with the theoretical mean of $150 \mathrm{sec}: t(29)=9.40, p<.0001]$. There was no effect of sex (data not shown). This spontaneous preference for the dry vial is not correlated with the avoidance score $(r=.16, n=30$, n.s.), which shows that for each fly the avoidance score does not mainly reflect the spontaneous aversion to humidity. In such conditions, one may conclude that the increased avoidance of the lighted vial during training is most probably due to learning.

\section{GENERAL DISCUSSION}

Experiment 1 confirmed that replacing the lighted vial containing a solution of quinine with a dry vial is sufficient to make flies choose the lighted vial after they have been trained to avoid it (Figure 2). Hence, flies are able to avoid the lighted vial containing a solution of quinine, but removing the quinine disrupts this avoidance. It could be that the absence of humidity is sufficient to disrupt the previously learned association or that humidity, detected at the choice point, is an unconditioned aversive stimulus that accounts at least in part for the avoidance of the lighted vial during training. In such conditions, an extinction process cannot be observed because a gradual fading of the avoidance is expected when the aversive stimulus is removed, at least during the first extinction trials.

It seems that in our paradigm photopositive flies learned that going toward light is associated with an aversive stimulus but, contrary to expectation, the main aversive stimulus is not tarsal contact with quinine. We may hypothesize that at the choice point of the T-maze the flies detected differences between the two arms other than the presence of quinine, which can be detected only after the lighted vial is reached. These differences could be linked to humidity (because only the lighted vial is wet) or to a possible repellent odor of quinine. Experiment 2 showed that the flies trained with quinine, but without tarsal contact with it, had higher avoidance scores than those trained with a lighted dry vial (see Figure 3). The flies thus avoided the lighted vial containing an aversive stimulus, which could have been the presence of humidity or a quinine odor. However, Experiment 3 showed that similar scores are obtained when flies are trained with humidity only or in a situation in which a potential quinine odor might be present (Figure 4A). Clearly, it seems that humidity is an aversive stimulus that is necessary and sufficient to induce the avoidance response in the maze and that flies do not perceive any quinine odor.

If humidity is an aversive stimulus, removing the opaque vial covering the darkened vial should be sufficient to in- duce a high avoidance of the vial containing quinine, because flies now have a choice between two equally lighted vials, one of which is paired with unpleasant stimuli (humidity and quinine). The flies did indeed choose the vial without such stimuli (Figure 4B).

Finally, it was of interest to know whether flies could be sensitized by quinine during training, which could explain why they avoid the lighted vial more when it contains quinine than when it contains water only (Figure 5B). Exposing flies to quinine for several hours before training had no effect on avoidance scores (Figure 5A). Therefore, it seems that quinine is actually an aversive stimulus to which flies do not become sensitized in our paradigm.

To sum up, these experiments show that, contrary to what was expected, the main aversive stimulus in our paradigm is not quinine but humidity. Flies crossing a maze toward light have to walk in a humid (unpleasant) area and on quinine. Consequently, they inhibit their photopositive tendency in order to avoid humidity and quinine. In such conditions, we can no longer consider (Le Bourg \& Buecher, 2002, p. 341) that humidity is a CS predicting a US (quinine) - humidity, like quinine, is a negative reinforcer. However, one cannot assert that humidity is more aversive than quinine, because flies perceive humidity before quinine and thus make an association between humidity and light before they encounter quinine. Moreover, the delay between light and punishment by quinine could explain why flies trained with quinine have scores that are not much higher than the scores of flies trained with water only (Figure 5B), because increasing the delay between the response and the negative reinforcer decreases learning scores (see Lieberman, 2000, for a review). Thus, even if humidity appears to be more important than quinine as an aversive stimulus in this paradigm, similar results are not expected in paradigms for which there is no such order of presentations of the negative reinforcers. It may be said that in our paradigm photopositive flies are subjected to an initial aversive stimulus (high relative humidity) while crossing the lighted arm and to a second (taste of quinine) when walking into the lighted vial. Le Bourg and Buecher (2002) reached another conclusion: Photopositive flies are subjected to an aversive stimulus (taste of quinine) when walking into the lighted vial and learn to use humidity as a CS to avoid the lighted vial. The present results show that this explanation is untenable.

However, it cannot be concluded from the series of experiments described in this article that no learning occurred in our paradigm. Indeed, it could be argued that flies simply avoid highly humid environments and that this is sufficient to explain the "learning" results, but some arguments contradict this hypothesis.

First, about $80 \%$ of the flies chose the lighted vial on the first trial, and the presence of quinine (and thus of water) in the lighted vial has no effect on this tendency (see Experiment 2). In other words, flies prefer light to dark even if they have to walk in a humid environment, which shows that the aversion to humidity is not of paramount importance on the first training trial. 
Second, avoidance of the lighted vial containing quinine or water increases over trials, which indicates that the preference for the lighted vial decreases with experience of the aversive stimuli encountered by flies - that is, a humid arm leading to a wet vial, which can also contain quinine. Flies thus face a conflict between their preference for light and their aversion to humidity, and they need several trials to (learn to?) avoid humidity. Figure 4B shows that when there is no such conflict between light and humidity flies have a greater preference for the dry vial. It could be argued that the increased avoidance of the lighted vial over trials is simply due to increased relative humidity at the choice point during training. This explanation can be ruled out because two mazes were used for each fly, one with the lighted vial on the left and one with the lighted vial on the right (see the General Learning Procedure). Thus, every two trials the mazes were alternated, allowing evaporation of the humidity in the other maze.

Third, the addition of quinine to the lighted vial (Figure 5B; Le Bourg, 2004, Experiment 2; Le Bourg \& Buecher, 2002, Experiment 1) increases avoidance of the lighted vial. This result indicates that this aversive stimulus, which unlike humidity is encountered only in the lighted vial and not at the choice point, decreases preference for the lighted vial. Such a result is expected if there is an operant association between an aversive stimulus (quinine) and a behavior (avoidance of the vial containing quinine).

Fourth, preexposure to humidity did not modify the avoidance score and individual aversion to humidity was not correlated with the individual avoidance score (Experiment 5). Therefore, flies do not avoid the lighted vial during training because they are sensitized to humidity; more probably, they learn to avoid it because it is associated with aversive stimuli (i.e., humidity and quinine). Moreover, with similar motivation levels - that is, the same aversion to humidity - some flies learn to avoid the lighted vial whereas others do not.

Considering all these results - low initial avoidance of humidity, increased avoidance of the wet lighted vial over trials, aversive effect of a stimulus (quinine) not perceived at the choice point, no correlation between aversion to humidity and avoidance score - one can conclude that our paradigm shows that flies learn to avoid an area associated with aversive stimuli.

These experiments show that stimuli usually considered as neutral or useful to flies, such as humidity, can serve as a US in a learning task. Obviously, since the flies live in vials in which the relative humidity is high (approximately $70 \%$ in the vials used here; Le Bourg \& Minois, 1999) and rapidly die in dry air (Minois \& Le Bourg, 1999), this result was not expected. In such conditions, flies subjected to learning procedures in which aqueous solutions are used, such as PER paradigms (see, e.g., Brigui et al., 1990; see McGuire, 1984, for a review), could be subjected to a US other than the one to which they are supposed to be subjected (quinine in PER stud- ies). In PER studies, fasting flies walk on revolving drums and are subjected to a strip soaked with a sucrose solution followed by another strip soaked with a quinine solution. Flies walk on a very wet drum in order to remove quinine and sucrose from tarsal chemoreceptors (see, e.g., Brigui et al., 1990) or cross a wet strip every minute (DeJianne, McGuire, \& Pruzan-Hotchkiss, 1985). The present results suggest that walking on wet ground could be an aversive stimulus. In fact, this result may not be so surprising. We may imagine that even if water is essential to life, nonaquatic animals are not prone to spend much time in wet environments. In human beings as in flies, water is essential, but very few of us enjoy singing in the rain.

\section{REFERENCES}

Ackerman, S. L., \& Siegel, R. W. (1986). Chemically reinforced conditioned courtship in Drosophila: Responses of wild-type and the dunce, amnesiac and don giovanni mutants. Journal of Neurogenetics, 3, 111-123.

Brigui, N., Le Bourg, E., \& Médioni, J. (1990). Conditioned suppression of the proboscis-extension response in young, middle-aged, and old Drosophila melanogaster flies: Acquisition and extinction. Journal of Comparative Psychology, 104, 289-296.

DeJianne, D., McGuire, T. R., \& Pruzan-Hotchiss, A. (1985). Conditioned suppression of proboscis extension in Drosophila melanogaster. Journal of Comparative Psychology, 99, 74-80.

LE Bourg, E. (2004). Effects of aging on learned suppression of photopositive tendencies in Drosophila melanogaster. Neurobiology of Aging, 25, 1241-1252.

Le Bourg, E., \& BADIA, J. (1995). Decline in photopositive tendencies with age in Drosophila melanogaster (Diptera: Drosophilidae). Journal of Insect Behavior, 8, 835-845.

Le Bourg, E., \& Buecher, C. (2002). Learned suppression of photopositive tendencies in Drosophila melanogaster. Animal Learning \& Behavior, 30, 330-341.

Le Bourg, E., \& Minois, N. (1999). A mild stress, hypergravity exposure, postpones behavioral aging in Drosophila melanogaster. Experimental Gerontology, 34, 157-172.

Lieberman, D. A. (2000). Learning: Behavior and cognition (3rd ed.). Belmont, CA: Wadsworth Thomson Learning.

McGuire, T. R. (1984). Learning in three species of Diptera: The blow fly Phormia regina, the fruit fly Drosophila melanogaster, and the house fly Musca domestica. Behavior Genetics, 14, 479-526.

MéDIONI, J. (1958). Le comportement de Drosophila melanogaster Meig. dans un appareil à choix lumineux: Étude comparative de souches sauvages de provenances géographiques diverses [The behavior of Drosophila melanogaster Meig. within a phototaxic testing apparatus: Comparison of various geographical strains]. Comptes Rendus de la Société de Biologie, 152, 1004-1007.

Minois, N., \& Le Bourg, E. (1999). Resistance to stress as a function of age in Drosophila melanogaster living in hypergravity. Mechanisms of Ageing \& Development, 109, 53-64.

QuinN, W. G., Harris, W. A., \& Benzer, S. (1974). Conditioned behavior in Drosophila melanogaster. Proceedings of the National Academy of Sciences, 71, 708-712.

SAYeEd, O., \& Benzer, S. (1996). Behavioral genetics of thermosensation and hygrosensation in Drosophila. Proceedings of the National Academy of Sciences, 93, 6079-6084.

Wolf, R., \& Heisenberg, M. (1991). Basic organization of operant behavior as revealed in Drosophila flight orientation. Journal of Comparative Physiology A, 169, 699-705.

(Manuscript received May 28, 2004; revision accepted for publication October 6, 2004.) 